\title{
DE 1390 À 1420, LE ROI CHARLES III DE NAVARRE PROTECTEUR DES JUIFS ÉTRANGERS À SON ROYAUME
}

BEATRICE LEROY

Université de Pau

\begin{abstract}
«... A Maestre Exdra Alazar, fisico, al quoal el Seynnor Rey informado e certifficado pleneramente de la sufficiencia e discrecion deill e de las buenas obras e practicas de medizinas que eill ha fecto tanto en su Regno como en otra part, entendiendo que deill puede ser bien servido, el dicto Seynnor Rey lo ha retenido por su fisigo. Et affinque aya con que mantener su estado, el dicto Seynnor Rey ha ordenado que eyll aya de aver deill cadaynno de gages ata tanto que el dicto Seynnor Rey aya otrament ordenado deill CXLIII ${ }^{\circ}$ florines por aynno, repartidos por ygoales pagas en cadauno de los meses del aynno, es assaber XII florines por mes a primero pagamento en el primero día de Jenero deste aynno $\mathrm{CCCCXV}^{\circ}$ e en adelant cada mes e en cadaun aynno durant la voluntat del dicto Seynnor Rey, segunt se contiene por su lettra data en Olit XVIII ${ }^{2}$ día de Jenero CCCCXV^..." '.
\end{abstract}

En 1415, Charles III de Navarre retient pour l'un de ses médecins personnels maitre Esdra Alazar, l'un des Juifs de Saragosse les plus connus dans ces années ${ }^{2}$. Cet évènement, si c'en est un, permet de réfléchir sur le règne de Charles III de Navarre (1387-1425), et sur le royaume de Navarre qui, dans ces années, semble ouvrir ses portes aux Juifs des royaumes voisins. Il nous est agréable de prolonger ainsi la réflexion amorcée il y a quelques années grâce à la revue Sefarad, à propos d'«accueil» ou de "tolérance" en Navarre ${ }^{3}$, et continuée trés récemment grâce à David Romano qui nous demanda,

1 AGN, Registros de Comptos (RC), t. 337, fol. 50.

2 A. Blasco Martinez, La judería de Zaragoza en el siglo XVI, Zaragoza 1988.

${ }^{3}$ B. LeroY, «Le royaume de Navarre et les Juifs aux XIVo et $\mathrm{XV}^{2}$ sizcles: entre l'accueil et la tolérance», Sefarad XXXVIII (1978) 263-292. 
à Lerida en novembre 1989, un aperçu sur les relations des Juifs de Navarre et des Juifs d'Aragon.

\section{LA NAVARRE, TERRE D'ACCUEIL POUR LES JUIFS}

Le royaume, gêré par la dynastie des comtes d'Évreux (puis ducs de Nemours) depuis 1328, surtout au temps de Charles II (1349-1387) et de Charles III, et qui compte de très intéressantes communautés juives ${ }^{4}$, semble suivre une politique très affirmée, l'accueil des Juifs qui pourraient être inquiétés dans les États voisins de la péninsule. Dès 1370, alors même que la "révolution Trastamare" pouvait mettre en péril les aljamas du diocèse de Calahorra, la reine Jeanne (de Valois, fille de Jean II le Bon) au nom de son mari Charles II, avait fait savoir à tous les Juifs de Castille attendant leur droit d'entrée à Calahorra, qu'ils pouvaient se présenter en Navarre, sans se soumettre à de trop fortes impositions communautaires, mais en payant une capitation de 2 florins au Trésor, ce qui était une très forte taxe d'invitation s. Mais les bons rapports se sont vite rétablis d'un royaume à l'autre, d'aljama en aljama, et surtout la paix est revenue dans les juderias de Castille sous Henri II de Trastamare malgré les troubles des débuts. Les grands seigneurs reprennent l'habitude de s'entourer de Juifs ou de se décharger sur eux pour leurs affaires. Très naturellement, les grands de Castille traitent avec les Juifs de Navarre lorsqu'ils ont des intérêts dans ce royaume, tel Juan Hurtado de Mendoza, vassal-lige de Charles III pour raisons diplomatiques. A partir de 1394, ce noble castillan nomme procureur en Navarre, pour administrer son fief, le Juif de Tudela Samuel Benvenist ${ }^{6}$. De son côte, l'épouse de Charles III, l'Infante Leonor de Trastamare, est venue en Navarre avec son médecin personnel le Juif de Tolède Jucef ben Wakkar, qui se marie et fait souche à Tudela?

${ }^{4}$ B. LeROY, The Jews in Navarre in the Late Middel Ages, Jerusalem 1985 et M. Garcia-Arenal - B. Leroy, Moros y judios en Navarra en la baja Edad Media, Madrid 1984.

5 J. VAldeón BARUQUe, Enrique II de Castilla, la guerra civil y la consolidación del régimen (1366-1371), Valladolid 1966. En 1370 en Navarre: AGN, Sección de comptos, documentos (Comptos), Caj. 26, $\mathrm{n}^{\mathrm{Q}} 12$.

${ }^{6}$ AGN, Comptos, 1394: Caj. 70, $\mathrm{n}^{\circ}$ 35, I et 1395: Caj. 78, $\mathrm{n}^{2} 44$, III.

${ }^{7}$ Entre autres, sur Josef ben Wakkar: en 1396, il arrive, est dit encore "médecin du roi de Castille», et se marie en Navarre (AGN, Comptos, Caj. 78, $\mathrm{n}^{\circ}$ 5, I), le roi lui offre 200 florins; en 1400: AGN, Comptos, Caj. 85, $\mathrm{n}^{\circ}$ 11, I, II, III; en 1412 il a un fils, Jacob (AGN, RC, t. 318, fol. 8). 
Avec l'Aragon, tout semble se dérouler très normalement dans la vie quotidienne, dans les affaires, dans les relations des Juifs de Navarre et des Juifs d'Aragon. Ainsi Samuel Amarillo de Tudela, qui dispose de trois montures et de deux aides, va très régulièrement de Tudela à Saragosse et de Tudela à la cour de Navarre, entre 1380 et 1390, pour approvisionner celle-ci en tout ce qui lui est nécessaire; le roi Charles II l'avait déjà chargé de missions secrètes, en 1382 par exemple ${ }^{8}$.

Or, dans cette atmosphère apparemment sereine, éclate l'année 1391, qui est si angoissante pour les Juifs de Castille et pour ceux d'Aragon, comme les années suivantes pendant lesquelles le calme ne peut regagner les esprits. En Navarre, aucun "émoi» n'a secoué les aljamas - le souverain y a certainement veillé- et dans les années suivantes Charles III reçoit tous les Juifs qui le désirent. Ce ne sont pas des foules de déplacés qui se présentent à la frontière; mais quelques personnalités et quelques dates méritent de retenir l'attention. En 1393, Samuel Benquis, Juif de Saragosse, paie 200 florins au Trésor de Navarre, car le roi lui a permis de s'installer chez lui ${ }^{\circ}$. En 1398, le couple royal demande au trésorier Juan Caritat de se charger des dépenses d'un homme de confiance, Geoffroy, qui est allé à quatre reprises en Béarn pour y négocier la délivrance de deux Juifs d'Aragon enlevés de chez eux et emmenés en Béarn "sous couleur de guerre» ${ }^{10}$. Entre temps, les Juifs ont été chassés du royaume de France; aucun d'entre eux ne semble avoir frappé aux portes de la Navarre, qui avait largement accueilli les Juifs français de 1306 et de 1322; ces derniers ont-ils choisi d'autres lieux d'exil, ou bien Charles III, cousin de Charles VI de France et assez actif dans les affaires françaises lorsqu'il se trouve à Paris auprès des ducs, a-t-il voulu éviter de se démarquer de la politique

${ }^{8}$ Entre autres, sur Samuel Amarillo: en 1382: AGN, Comptos, Caj. 43, $\mathrm{n}^{2}$ 18, v: «...por mano de Simuel Amariello Judio de Tudella, por mandamiento a eill fecho de boca, el $\mathrm{VIII}^{\circ}$ dia de octobre postremeramente passado, es assaber a dos ciertas personas a las quales lis fiziemos dar, e por ciertos grandes servicios que fechos nos avian, Cient e Veynte Dos francos doro" En 1388: AGN, Comptos, caj. 54, $n^{2} 27, x$, $\mathrm{XI}$, Samuel souscrivant en caractères hébraïques et apposant son sceau personnel.

9 AGN, RC, t. 219, fol. 7: «... de Simuel Venquis, Judio de Çaragoça, por cierto privilegio e franqueza que el Rey le ha otorgado por fincar en el Regno, IX ${ }^{2}$ dia de março, II $^{c}$ florines, vallen II $^{c} L X 1 \ldots$ Des Venquis sont déjà installés en Navarre; en 1391, Gento Venquis est collecteur de la aljama du Val de Funes: AGN, Comptos, Caj. 62, $\mathrm{n}^{2} 19, \mathrm{XXXV}$.

10 AGN, Comptos, Caj. 76, n 34, IV. 
des Valois? Quoi qu'il en soit, en 1398 Charles III voyage en France accompagné de son médecin personnel le grand rabbin de Navarre Josef Orabuena ".

En 1402, Charles III reçoit à Sangüesa et à Tudela Hasdaï Crescas, pour affaires secrètes, pour affaires personnelles, répètent les documents officiels. Le grand intellectuel de Saragosse (qui souscrit ses actes et quittances en caractères hébraïques, et que le rabbin de Tudela était venu rencontrer en 1401 personnellement à Saragosse), est donc venu auprès du roi de Navarre traîter des affaires importantes, tenues cachées quelque temps. Il est très probable que Crescas s'est déplacé pour acheter une sorte de droit de protection en Navarre, pour ses Juifs aragonais mal remis des tueries et des conversions forcées de 1391. En effet, en 1412 (installation sur le trône d'Aragon de Fernando de Antequera, et début de la Dispute de Tortosa), des Juifs d'Aragon se présentent en Navarre "pour cause de mortalité» dans leur royaume (?) et Charles III les fait installer à Tudela... moyennant 900 florins fort bien venus pour aider à la réfection du grand château royal de la ville ${ }^{12}$.

C'est ainsi qu'en 1414 (à la fin de la Dispute de Tortosa) maître Esdra Alazar est invité en Navarre pour y soigner l'Infante Dona Johanna ${ }^{13}$; on sait que dans l'année 1415, il est retenu au service de Charles III. Cette politique particulière est connue dans la péninsule; en 1418, Charles III permet à Maître Samuel de Grenade de s'installer

11 AGN, RC, t. 243 (année 1398), fols. 33-39 et 90-92.

12 AGN, RC, t. 263 (année 1401), fol. 52: «... A Maestre Astruc, Rabi de la Aljama de los Judios de Tudella, por fazer sus expensas a yr en Çaragoça con maestre Azday por ciertas cosas que el Rey encargado et mandado le avia, segunt se contiene por mandamiento data $\mathrm{XIII}^{\circ}$ dia de Mayo anno $\mathrm{CCCC}^{\mathrm{mo}}$ Primo (...) pagado por el dicto Juce Horabuena, Ve Florines, valen VI 1. XIII s. III d.". 1412: t. 318, fol. 28. En 1402: AGN, Comptos, Caj. 87, $\mathrm{n}^{\circ}$ 16, IV: “... Sepan todos que Yo, Maestre Azday, Rab de los Judios de Çaragoça, otorgo aver avido e recebido de Vos Abram Enxoep, Recebidor de la Merindat d' Estella, los quales el seynnor Rey me los mando dar, por mis expensas de yr a Sanguesa, a Exea, e otras partes por su servicio e por ciertos mesageros que yo he imbiado de las dictas partidas enta el, la suma de quoaranta florines d'Aragon, que a XXV s. XVIII d. pieza valen Sesanta e Seys libras Treze sueldos Quatro dineros. De la quoal dita suma me tiengo por bien pagado por testimonio de las presentes firmadas de mi nombre con mi propria mano. Data en Olit XVIII ${ }^{\circ}$ dia de marzo aynno de Mil IIIIc e dos ... Azday Cresgus" (et en caractères hébrailques, Hasdal Crescas).

13 AGN, RC, t. 333, fol. 34: «... A Ezdra Alazar de Çaragoça, fisico, pora fazer sus expensas a yr a la dita Dona Johanna do el Rey lo imbio, por regir e curar de cierta enfermedat que eilla ha, $X$ florines que a XXIX $s$. pieça contenido en el dito mandamiento, valen XIIII 1. X s.". 
chez lui ${ }^{14}$. Si bien qu'à la fin du siècle, lorsque les conversos de Saragosse Sánchez et Santángel, compromis dans l'assassinat de Pedro de Arbués, cherchent un refuge, c'est tout naturellement en Navarre qu'ils se précipitent.

\section{LA POLITIQUE DU ROI DE NAVARRE}

Cependant, les souverains de Navarre sont des rois chrétiens, et qui tiennent à la qualité de leur administration; ce sont là vérités premières, mais qui expliquent quelques cas d'intransigeance et de prosélytisme. Charles III en 1390 a nommé Grand Rabbin de Navarre son médecin juif personnel, Josef Orabuena, un Juif de Tudela, désormais rémunéré de très belles rentes assises sur divers bâtiments du fisc de Tudela, Estella et Pampelune, et très attaché à la personne royale. On sait qu'il voyage avec le roi, même en France, qu'il lui prête constamment de grosses sommes sur ses biens personnels ou qu'il trouve à son intention auprès des Juifs de Navarre, comme par exemple en 1400 auprès du receveur Abraham ben Shuaib ${ }^{13}$, ou auprès des négociants de Barcelone au hasard de voyages. Maître Josef peut se faire aider de ses fils, Itzhak, Leon, Abraham et Jeuda, qui lui succèdent dans les affaires avec la cour à sa mort en 1416. Or, Grand Rabbin et donc porte-parole de ses coreligionnaires auprès du trône, et du gouvernement auprès de ses Juifs, il est constamment occupé à racheter ou plutôt faire racheter par divers condamnés, de très lourdes amendes. Même Nathan del Gabbaï et Samuel Amarillo les indispensables fournisseurs et prêteurs, sont condamnés à verser des sommes, pour de quelconques abus, en 1392 et $1393{ }^{16}$.

14 AGN, Comptos, Caj. 119, ne 3: “... Como maestre Simuel de Granada, Judio, venido de tierras extranias en nuestro Regno ya proposito de continuar en nuestro servicio por algunas cosas que mandado li avemos, Nos, queriendo proveyr de su provision e mentenimiento en tal que meror afeccion pueda usar en lo que encargado li avemos ...".

is AGN, Comptos, Caj. 79, n 13, XLIX: «...Seppan todos que yo Abraham Enxoep, Judio, otorgo aver ovido e recebido de Johan Karitat thesorero de Navarra, por pagar la husura de $\mathrm{II}^{\mathrm{c}}$ florines que por servicio del Rey tome a mailleuta, delivrado a mi por Maestre Juce Horabuena, la summa de Veynte florines que a XXVI s. VIII d. pieça valen Veynte Seys libras Treze sueldos Quoatro dineros gros en II s. De la quoal dicta summa de XXVI 1. XIII s. III d. me tengo por bien pagado...". En 1389, Josef Orabuena, à titre de médecin du roi, perçoit 50 livres par an sur la teinturerie d'Estella: AGN, Comptos, Caj. 57, n² 8, II.

16 AGN, RC, t. 219, pour l'anncé 1393. 
Ces mêmes années d'accueil de la part du roi de Navarre, sont aussi des moments de grands jugements d'officiers juifs convaincus de malversation dans leur gestion. En 1388, Jeuda Levi d'Estella, receveur-collecteur de sa merindad, est condamné à verser une forte somme, sur ses biens saisis, inventoriés et négociés jusqu'à concurrence de l'amende demandée. En 1411; Abraham ben Shuaib, qui fut aussi depuis le début du XV $\mathrm{XV}^{2}$ siècle receveur-collecteur des Montagnes d'Estella, malgré sa position familiale de gendre du Grand Rabbin Orabuena, est condamné et exécuté par pendaison, tous biens saisis et réunis au Trésor " $"$.

Mais ces condamnations, rachetées ou non, sont actes de souveraineté et non pas du tout témoignages d'antijudaïsme. Il est également dangereux de vouloir interprêter trop loin des cas de conversion. En 1392, Charles III est le parrain de quelques Juifs qui se sont faits baptiser à Olite, à la fête de Noël; en 1412, il offre un pourpoint à un certain Gabriel de Viana qui est entré dans le christianisme ${ }^{18}$. Les conversions sont rares en Navarre; il y en a tout de même, de temps à autre. Ces deux dates de 1392, au lendemain des terreurs de 1391 dans les royaumes voisins, et de 1412, qui est une année inquiétante dans la couronne d'Aragon, sont intéressantes à relever pour une histoire des conversos. Charles III ne peut que parrainer et récompenser des Juifs qui se convertissent au christianisme. Mais on n'assiste jamais en Navarre à des conversions massives, sous le poids d'une émeute ou d'une prédication populaire, du moins pas depuis la matanza de 1328 et pas avant l'exil de 1498, en tous cas pas durant le règne de Charles III.

Celui-ci, comme déjà l'a fait son père Charles II, permet un plein épanouissement des aljamas navarraises. Les Juifs ont le droit de posséder des propriétés, en ville et dans les campagnes. Les biens inventoriés de Jeuda Levi et d'Abraham ben Shuaib montrent l'é-

17 B. Leroy, "Les comptes d'Abraham Enxoep au début du XV siècle", Principe de Viana, 38 (1977), 177-205; IDEM, «De l'activité d'un Juif de Navarre, fin du $14 e$ siècle», Archives Juives, 17e année, (1981) 1-6. Les Juifs peuvent également exécuter leur "malsin». Ceci s'est rarement produit, mais on en a tout de même quelques traces en Navarre: en 1413, Samuel Maños d'Estella, AGN, Comptos, Caj. 102, n 5 , $v$, et en 1421, Sento Jaura de Pampelune, exécuté à Puente-la-Reyna: AGN, RC, t. 365, fols. 3-4.

18 1392, AGN, RC, t. 216 (année 1392), fol. 111: «... A Menauton de Bayonna, por paynnos, foraduras, jupones e tella comprados pora bestir los ditos conversos que fueron Judios e fueron baptizados en Ollit el dia de Nadall LXXXXII, de que el Rey fue padrino...". En 1412, Gabriel de Viana, AGN, RC, t. 318, fol. 139. 
tendue et la complémentarité de leurs terres et jardins dans la région d'Estella. On peut en dire autant si ce n'est mieux des Juifs de Tudela, propriétaires dans toute la huerta de l'Èbre; comme des Juifs des villages de la vallée, qui, en petites communautés, vivent d'une vie paysanne en plus de leurs occupations traditionnelles artisanales et financières ${ }^{19}$. A Pampelune, les Juifs habitant une judería essentiellement urbaine, n'ont pas de place dans les campagnes. De 1390 à 1415, une génération de très grandes personnalités juives entoure la famille royale, occupe des emplois dans la fiscalité, prête au roi et aux grands autant qu'à toutes les sociétés rurales et urbaines, comme c'est le rôle traditionnellement reconnu aux Juifs. Ces hommes sont aussi les diplomates secrets ou officiels du souverain, lorsqu'ils vont en son nom à Saragosse (en 1401 par exemple) ou ailleurs. Ils sont hommes du pouvoir, hommes de la cour. De 1385 à 1420 environ, habitude qui devient presque essentielle à la bonne marche du royaume, ils sont arrendadores des impôts de Navarre, en équipes de six à dix-huit Juifs ou Chrétiens et Juifs mélangés, selon les années, versant des fermes annuelles de 60.000 à 100.000 livres de Navarre. Parmi eux, Jeuda Levi a pu se rétablir de sa saisie de 1388, et Abraham ben Shuaib, a été très actif jusqu'en 1410. Les principaux sont Josef Orabuena, Samuel Amarillo, Samuel Benveniste, Nathan Del Gabbay, Jeuda ben Menir, tous ceux-ci de Tudela, et puis Ezmel Even David d'Olite, Itzhak Medellin d'Estella, plus rarement Itzhak Alborge de Pampelune ${ }^{20}$.

La cour de Navarre ne se passerait pas de techniciens juifs. Ceux-ci, quel que soit leur emploi par ailleurs, négocient et relient les livres. En 1392, Charles III achète pour 25 livres de Navarre au Juif Samuel «el Corretor" un bréviaire... "de la Règle de Rome»

19 B. LEROY, "Les Juifs et le monde rural dans la vallée moyenne de l'Èbre, dans la deuxième moitié du XIV siècle", in Ninth World Congress of Jewis Studies, vol. I, Jerusalem 1986, pp. 83-90.

La controverse entre David Romano et nous, est ouverte. Les Juifs de la région évoquée sont-ils uniquement habitants des villes?

${ }^{20}$ B. LEROY, "Les Juifs de Navarre et les pouvoirs pendant les règnes de Charles II et Charles III (1349-1425)", in Minorités et marginaux en France méridionale et dans la péninsule Iberique (VIIe-XVIIle siècles), ed. CNRS, Paris 1986, pp. 157-176; IDEM, "Les hommes du pouvoir en Navarre au XIV ${ }^{2}$ siècle. Gouvernement et société dans le royaume de Navarre de 1328 à 1425", in Le Moyen Age XCV, 1989, 475-490; IDEM, "La cour des rois Charles II et Charles III de Navarre (vers 1350-1425), lieu de rencontre, milieu de gouvernement» in A. RucQuor (dir.), Realidad e imágenes del poder, Valladolid 1988, pp. 233-248. 
qu'il fait offrir à Johaneta fille de son valet de chambre et damoiselle de l'Infante Johanna. Le roi fait également donner cinq sous (c'est peu... pour un petit travail?) au rabbin de Tudela qui a relié, ou fait relier, le «Roman de Lancelot" prêté à messire Lionel, son fils bâtard, pour qu'il y apprenne à lire ${ }^{21}$. Les pelletiers, merciers, orfèvres, couturiers, ou encore les marchands de chevaux, Juifs de Navarre, son très nombreux dans l'entourage royal. Grands négociants et courtiers en affaires fournissant tout à la commande, ou travailleurs de leurs propres mains, voici par exemple en 1415, les couturiers et pelletiers Juce Cayat, Gento Alfaça, Abraham Cortes, de Pampelune, les tondeurs de drap Salomon d'Olite et Mosse Aliamin, le teinturier Azach Melech, le savetier Abraham Adida, les argentiers-orfèvres Samuel Ezquerra, Abraham Almaquarel, Samuel Levi, et puis encore les marchands de drap Juce Levi, Abraham La Rabiça, Saül Levi, Juce Biel, Matascon, Azach Evendavid, Alazar Levi; Jacob Cohen le mercier de Saragosse et maitre Leon Orabuena ont fourni des fils d'or, des crespines, et de la fourrure de menu vair. Enfin, Jeuda Orabuena, le médecin du roi à la suite de son père, a permis au roi d'acheter une pierre précieuse à un Juif d'Aragon ${ }^{22}$.

Enfin, les rois de Navarre ont des médecins juifs, comme les Orabuena père et fils, comme Abraham Cominet retenu en même temps qu'Alazar, à 300 florins de gages annuels, comme les ben Wakkar, et plusieurs autres encore ${ }^{23}$. En 1414-1415, le roi Charles III avait-il besoin de retenir en plus à son service maître Esdra Alazar de Saragosse? Le texte le dit, la notoriété et l'extrême habileté du médecin Alazar expliqueraient le geste royal. Ce que le texte ne dit pas mais ce que disent tous les documents contemporains, est que les Juifs vivent protégés en Navarre et que, en période d'inquiétude à Saragosse,

${ }^{21}$ AGN, Comptos, Caj. 61, $\mathrm{n}^{2} 2$, XII, et Caj. 61, $\mathrm{n}^{2} 4$, IV.

22 B. LeRoY, «La cour des rois de Navarre dans la deuxième moité du XIVe siècle et au début du XVe siècle, une rencontre de techniciens", Anuario de Estudios Medievales 16 (1986), 307-318; 1415: AGN, RC, t. 337, fols. 13 à 177, dont fol. 100 par exemple: “... A Judas Orabuena, fisigo del Rey, por una piedra preciosa dicta sineralda engastonada en un anieillo doro que el seynnor Rey ha fecto comprar por eill de un Judio d'Aragon" (= 60 florins-or, ou 87 livres de Navarre à 29 s. piece).

${ }^{23}$ Tel maitre Bernart Cavailleria (un patronyme intéressant, mais le document navarrais ne dit pas s'il est converso ou non), en 1397, citoyen de Gerona, maitre médecin et licencié en Arts, qui a rejoint Charles III lors de son voyage en France, avec tous ses livres est-il bien précisé: AGN, Comptos, Caj. 71, $\mathrm{n}^{0} 3$, XVII et XVIII. 
il est facile de voyager quelques heures en remontant l'Èbre et de s'installer à Tudela. Il faut payer le roi de Navarre. Charles III, roi d'un État ibérique mais de famille française, fait acheter son droit d'accueil et de protection. Il tient à une politique très personnelle visà-vis des autres souverains ibériques et ne laisse pas empiéter sur ses pouvoirs. Libre à lui d'accueillir chez lui les Juifs inquiétés ailleurs. Libre à lui de leur emprunter à usure, et de leur laisser pratiquer tout ce qu'ils veulent. Mais il peut aussi les aider à se convertir; il peut enfin s'en faire payer, et très cher, et les condamner au besoin. Sa politique vis-à-vis de ses Juifs est l'une des manifestations de sa souveraineté et de sa liberté de faits et gestes. Le roi de Navarre ne doit pas laisser dicter sa politique par ses contemporains.

\section{RESUMEN}

El rey de Navarra Carlos III, como manifestación de su independencia y de su política individual, acogía en su reino a judíos extranjeros. En los años 1401-1402 recibió en Sangüesa a Hasdai Crescas, de Zaragoza, para tratar del asentamiento en Tudela de varias familias judías procedentes de aquella ciudad. El rey invitó también al Maestre Ezdra Alazar, físico de Zaragoza, manteniendo a su servicio al médico Josef ben Wakkar de Castilla. Este hecho se explica por la política de Carlos III de proteger las aljamas y dar cargos de responsabilidad a la elite de los judíos, al mismo tiempo que les invitaba a convertirse.

\section{SUMMARY}

As a manifestation of his independent frame of mind and individualistic policy, Charles IIl, King of Navarre used to welcome Jewish foreigners in his kingdom. In 14011402 he received Hasdai Crescas, of Zaragoza, in Sangluesa, to talk about the settlement in Tudela of some Zaragozan Jewish families. The King also invited Maestre Ezdra Alazar, a physician of Zaragoza, keeping in his service doctor Josef ben Wakkar of Castilla as well. This fact is explained through Charles III's general policy of protecting the aljamas and promoting the élite of the Jews to higher responsibilities, while leading them into conversion. 